\title{
Comparison of electron transport calculations in warm dense matter using the Ziman formula
}

\author{
D. J. Burrill, ${ }^{1}$ D. V. Feinblum, ${ }^{1,2}$ M. R. J. Charest, ${ }^{1}$ and C. E. Starrett $^{1, *}$ \\ ${ }^{1}$ Los Alamos National Laboratory, P.O. Box 1663, Los Alamos, NM 87545, U.S.A. \\ ${ }^{2}$ Department of Chemistry, University of California, Irvine, CA 92697, U.S.A.
}

(Dated: December 4, 2015)

\begin{abstract}
The Ziman formulation of electrical conductivity is tested in warm and hot dense matter using the pseudo-atom molecular dynamics method. Several implementation options that have been widely used in the literature are systematically tested through a comparison to accurate but expensive Kohn-Sham density functional theory molecular dynamics (KS-DFT-MD) calculations. The comparison is made for several elements and mixtures and for a wide range of temperatures and densities, and reveals a preferred method that generally gives very good agreement with the KSDFT-MD results, but at a fraction of the computational cost.

Keywords: electron conductivity, warm dense matter, average atom model, Ziman formula
\end{abstract}

\section{INTRODUCTION}

The calculation of electron transport coefficients in warm and hot dense matter has been the subject of a considerable amount of recent theoretical interest [1-7] due in part to the importance of such quantities for inertial confinement fusion modeling $[4,8]$. A lack of direct experimental data for electrical and thermal conductivities also drives the need for accurate and reliable theoretical calculations.

Kohn-Sham density functional theory molecular dynamics (KS-DFT-MD) has emerged as a powerful and accurate tool for simulating warm dense matter. By coupling it with the Kubo-Greenwood formulation [9-12] it has also been used to generate conductivity data. Unfortunately, the method is extremely computationally expensive, and for this reason is typically limited to low temperatures (a few tens of eV).

A much more computationally efficient alternative to KS-DFT-MD are average atom models. These come in many varieties [13-19] and are also density functional theory based models. The common theme to these models is that one tries to find the properties of one atom in the plasma. Average atom models have also been used to generate electronic transport coefficients. Broadly speaking, there are to two main approaches: on the one hand are Kubo-Greenwood based calculations [7, 20-22] that are qualitatively similar to the KS-DFT-MD calculations; and on the other hand are Ziman-type methods that estimate conductivity on the basis of electron-ion scattering cross sections [1, 13, 19, 23-25].

Previously [7], the average atom Kubo-Greenwood approach was systematically compared to KS-DFT-MD data for a number of elements and mixtures for a range of temperatures and densities. The comparison revealed reasonable agreement in some cases, but some serious disagreement in others, both qualitative and quantitative.

*Electronic address: starrett@lanl.gov
In this paper we adopt a similar systematic approach but using the Ziman-type method. While much has been published using this approach, a systematic evaluation of its accuracy relative to KS-DFT-MD simulations has not, to the best of our knowledge, been carried out. Moreover, to generate the inputs (i.e. scattering potential, ionization fraction and ion-ion structure factor) for the Ziman-type approach we use the recently developed pseudo-atom molecular dynamics (PAMD) method [26]. PAMD has been shown to be of comparable accuracy to DFT-MD methods for equation of state, ionic diffusion coefficients and ion-ion structure factors [26, 27], though it has limitations [28]. The advantage of PAMD over DFT-MD methods is that it is much less computationally expensive. As a result of the accuracy of PAMD, the comparison of electron conductivities to KS-DFTMD should be a fair test of the conductivity model (i.e. the Ziman-type formula), and not be seriously limited by inaccuracies in the underlying model.

In section II we review the Ziman-Evans formula for electronic conductivity, and then rewrite it in terms of an electronic relaxation time. This allows the connection and relationship of the Ziman-Evans formula to the recently developed expression of Johnson [20] to be clarified. Moreover, we are able to generalize Johnson's expression to explicitly include the ion-ion structure factor. We then give the extensions of these formulas for mixtures, and in the case of the Johnson formula, its extension to thermal and optical conductivities. In section III we summarize the pseudo-atom molecular dynamics method and discuss the options for coupling it to the conductivity formulas. Then in section IV the systematic comparison to the KS-DFT-MD data is carried out. We also test the accuracy of Thomas-Fermi PAMD compared to Kohn-Sham PAMD for warm dense aluminum. Finally in section $\mathrm{V}$ we draw our conclusions. 


\section{EXPRESSIONS FOR ELECTRON TRANSPORT COEFFICIENTS}

In this section we review the commonly used ZimanEvans formula for resistivity. We rewrite it in terms of an electronic relaxation time in line with Ziman's original derivation, and give the relationship between the direct approach to the conductivity (as originally proposed by Ziman [29]) and the inverse approach, where the inverse conductivity (i.e. the resistivity) is directly calculated (as proposed by Evans [30]). Both approaches have recently been used in the literature (eg. by Johnson [20] and Faussurier et al [1]) without discussion of their relative merits or relationship. Next we show how these formulations are easily extended to mixtures and finally, how the direct approach is easily extended to optical conductivity and thermal conductivity. We use Hartree atomic units in which $\hbar=m_{e}=e=1$.

\section{A. Ziman-Evans formulation}

The Ziman-Evans formula for calculating the resistivity $R$, which is the inverse of the conductivity, is $[13,30,31]$

$$
R=-\frac{n_{I}^{0}}{3 \pi\left(n_{e}^{*}\right)^{2}} \int_{0}^{\infty} d \epsilon \frac{\partial f}{\partial \epsilon} \int_{0}^{2 p} q^{3} \frac{\partial \sigma(\epsilon, \theta)}{\partial \theta} S_{I I}(q) d q
$$

where $\epsilon=p^{2} / 2, n_{e}^{*}$ is the number density of scattering electrons, $n_{I}^{0}$ is the density of ions, $S_{I I}(k)$ is the ion-ion static structure factor,

$$
q^{2}=2 p^{2}[1-\cos \theta]
$$

is the magnitude of momentum transfer squared, and

$$
f=\frac{1}{\exp \left(\beta\left(\epsilon-\mu_{e}^{*}\right)\right)+1}
$$

is the Fermi-Dirac occupation factor. Here $\beta=1 / T$ is the inverse temperature and $\mu_{e}^{*}$ is the electron chemical potential. The differential cross section is given by

$$
\frac{\partial \sigma(\epsilon, \theta)}{\partial \theta}=\frac{1}{p^{2}}\left|\sum_{l=0}^{\infty}(2 l+1) \sin \eta_{l} e^{\imath \eta_{l}} P_{l}(\cos \theta)\right|^{2}
$$

where $\eta_{l}$ are phase shifts and $P_{l}(\cos \theta)$ are Legendre polynomials. Equation (4) is referred to as the transition- or $t$-matrix approach to the cross section. The phase shifts $\eta_{l}$ in equation (4) are determined by solving the Schroedinger equation for a scattering potential $V^{\text {scatt }}(r)$.

In the Born approximation to the differential cross section we have

$$
\frac{\partial \sigma(\epsilon, \theta)}{\partial \theta} \approx \frac{1}{4 \pi^{2}} \tilde{V}^{s c a t t}(q)^{2}
$$

Using this approximation and integrating Eq. (1) by parts gives [32]

$$
R=\frac{n_{I}^{0}}{12 \pi^{3}\left(n_{e}^{*}\right)^{2}} \int_{0}^{\infty} d k k^{3} f(k / 2) \tilde{V}^{s c a t t}(k)^{2} S_{I I}(k)
$$

where $\tilde{V}^{\text {scatt }}(k)$ is the Fourier transform of $V^{\text {scatt }}(r)$.

\section{B. Relaxation time formulation}

Following Ziman [29] and Johnson [20] we define a relaxation time $\tau_{p}$

$$
\frac{1}{\tau_{p}}=\pi n_{I}^{0} \frac{v}{p^{4}} \int_{0}^{2 p} d q q^{3} \frac{d \sigma}{d \theta}(p, \theta) S_{I I}(q)
$$

where $p=m_{e} v$. This agrees with the relaxation time used in [20] except that we have included a structure factor, whereas Johnson's formula has $S_{I I}(q)=1$. We can now write down the conductivity $\sigma_{D C}$ using the Boltzmann approach of Ziman [29] and the resistivity $R$ using $[30]$

$$
\begin{gathered}
\sigma_{D C}=-\frac{1}{3 \pi^{2}} \int_{0}^{\infty} d \epsilon \frac{d f}{d \epsilon} v^{3} \tau_{p} \\
R=-\frac{1}{3 \pi^{2}\left(n_{e}^{*}\right)^{2}} \int_{0}^{\infty} d \epsilon \frac{d f}{d \epsilon} v^{3} \frac{1}{\tau_{p}}
\end{gathered}
$$

As $T \rightarrow 0, d f / d \epsilon \rightarrow-\delta\left(\epsilon-\epsilon_{F}\right)$, where $\epsilon_{F}$ is the Fermi energy. In this limit the Fermi velocity $v_{F}=\left(3 \pi^{2} n_{e}^{*}\right)^{1 / 3}$ and both these expressions give the same result

$$
\sigma_{D C}=\frac{1}{R}=n_{e}^{*} \tau_{v_{F}}
$$

i.e. the Drude form. For $T>0$ however, the results will differ. We refer to equation (8) as the direct approach to the conductivity, and equation (9) as the inverse approach. It is easy to show that the inverse approach and the Ziman-Evans formula (equation (1)) are the same.

In recovering the Drude expression (equation (10)) from both formulations we have assumed that the chemical potential and electron density are related through

$$
n_{e}^{*}=c_{T F} I_{1 / 2}\left[\beta \mu_{e}^{*}\right]=\frac{1}{\pi^{2}} \int_{0}^{\infty} d \epsilon v f(\epsilon)
$$

where $c_{T F}$ is a constant [27] and $I_{1 / 2}$ is the Fermi integral of order 1/2 [27]. These equations (8), (9) and (11) implicitly assume a free electron density of states.

\section{Extension to mixtures}

To extend the Ziman-Evans formulation to mixtures we follow ref. [33]. The extension is simply achieved by 
the replacement:

$$
\frac{\partial \sigma(\epsilon, \theta)}{\partial \theta} S_{I I}(q) \rightarrow \sum_{i}^{N_{s}} \sum_{j}^{N_{s}}\left(x_{i} x_{j}\right)^{\frac{1}{2}} S_{i j}(q) \mathcal{F}_{i}(\epsilon, \theta) \mathcal{F}_{j}^{\star}(\epsilon, \theta)
$$

where $N_{s}$ is the number species and $\mathcal{F}_{i}$ is the scattering amplitude for species $i$

$$
\mathcal{F}_{i}(\epsilon, \theta)=\frac{1}{p} \sum_{l=0}^{\infty}(2 l+1) \sin \eta_{l}^{i} e^{\imath \eta_{l}^{i}} P_{l}(\cos \theta)
$$

or, in the Born approximation

$$
\mathcal{F}_{i}(\epsilon, \theta)=\frac{V_{i}^{\text {scatt }}(q)}{2 \pi}
$$

$x_{i}$ is the number fraction of species $i$ and $S_{i j}$ are the partial structure factors.

\section{Extensions to the direct approach}

Ziman [34] (Ziman's equation 9.9.7) gives the expression for conductivity in terms of the integral expressions

$$
K_{n}=-\frac{1}{3 \pi^{2}} \int_{0}^{\infty} d \epsilon \frac{d f}{d \epsilon}\left(\epsilon-\mu_{e}^{*}\right)^{n} v^{3} \tau_{p}
$$

so that $\sigma_{D C}=K_{0}$. He also gives the expression for the thermal conductivity $\kappa$ in terms of these integrals

$$
\kappa=\frac{1}{T}\left(K_{2}-\frac{\left(K_{1}\right)^{2}}{K_{0}}\right)
$$

For completeness we also give the extension of equation (8) to non-zero frequencies, but do not present any calculations based on it. Following Johnson [20],

$$
\sigma(\omega)=-\frac{1}{3 \pi^{2}} \int_{0}^{\infty} d \epsilon \frac{d f}{d \epsilon} v^{3} \frac{\tau_{p}}{\left(\omega \tau_{p}\right)^{2}+1}
$$

This gives the free-free contribution to the optical conductivity and uses a low-frequency approximation. This formula satisfies the conductivity sum rule

$$
\begin{aligned}
\int_{0}^{\infty} d \omega \sigma(\omega) & =\frac{1}{2 \pi} \int_{0}^{\infty} d \epsilon v f(\epsilon) \\
& =\frac{\pi}{2} n_{e}^{*}
\end{aligned}
$$

To our knowledge, such extensions have not been developed for the inverse approach, though an expression for the inverse thermal conductivity in the Born approximation has been given in ref. [32].

\section{E. Physical interpretation of the quantities in the Ziman-like equations}

The above Ziman-like equations (8) and (9) for resistivity require input of the potential from which the electron

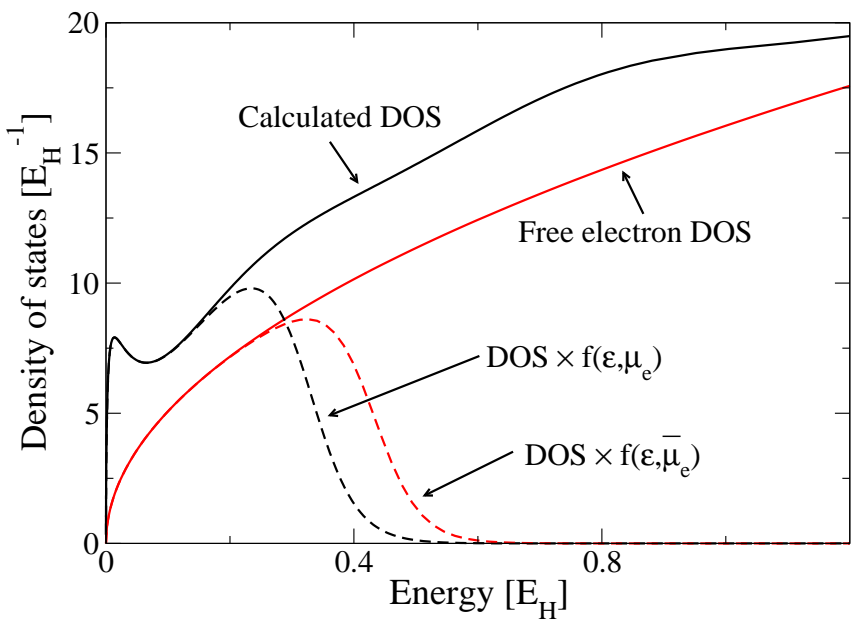

FIG. 1: (Color online) An example of a calculated density of states (DOS) compared to a free electron DOS for aluminum at solid density and $1 \mathrm{eV}$. The dashed lines are the "filled" states, i.e. the DOS multiplied by the Fermi-Dirac occupation factor. The area under either dashed curve is the number of ionized electrons per atom, both curves integrate to 3 , as expected for aluminum under these conditions. The conductivity formulas (equations (8) and (9)) assume a free electron density of states.

scatters $V^{\text {scatt }}(r)$, the density of scatterers $n_{e}^{*}$, the ion-ion structure factor $S_{I I}(k)$ and the electronic chemical potential $\mu_{e}^{*}$. In Ziman's original derivation [29], $V^{\text {scatt }}(r)$ is a weak pseudopotential such that the Born approximation is valid and the total potential $V^{t o t}(\boldsymbol{r})$ is given by the superposition of $V^{\text {scatt }}(r)$ at each atomic site, i.e.

$$
V^{t o t}(\boldsymbol{r})=\sum_{i} V^{\text {scatt }}\left(\left|\boldsymbol{r}-\boldsymbol{R}_{i}\right|\right)
$$

and $n_{e}^{*}$ is the density of valence electrons. Evans [30] assumed the same form (19) but $V^{\text {scatt }}(r)$ was allowed to be a strong scattering potential, not a pseudopotential. He further required that the scattering potential be of muffin-tin type, such that $V^{\text {scatt }}(r)=0$ for $r>R_{M T}$, where $R_{M T}$ is some muffin-tin radius. Formally, it is required that no two muffin-tin potentials overlap. The pseudopotential in Ziman's formulation is supposed to preserve the scattering properties of the full potential in Evans' approach.

For $n_{e}^{*}$ there are at least two reasonable choices. If the number of ionized electrons per ion is $\bar{Z}$, then the density of these valence electron is $\bar{n}_{e}^{0}=n_{I}^{0} \bar{Z}$. It seems reasonable to identify $n_{e}^{*}=\bar{n}_{e}^{0}$. However, the electron density $n_{e}^{*}$ is also related to the chemical potential $\mu_{e}^{*}$. If we take the limit $T \rightarrow 0$ of the conductivity formulas (equations (8) and (9)), we should recover the Drude form, equation (10). Because of the implicit free-electron density of states (DOS) in equations (8) and (9), to recover the Drude form $\mu_{e}^{*}$ must be related to $n_{e}^{*}$ by equation (11). If $n_{e}^{*}=\bar{n}_{e}^{0}$ then we can use equation (11) to find a corresponding chemical potential, $\bar{\mu}_{e}$. However, in general the 


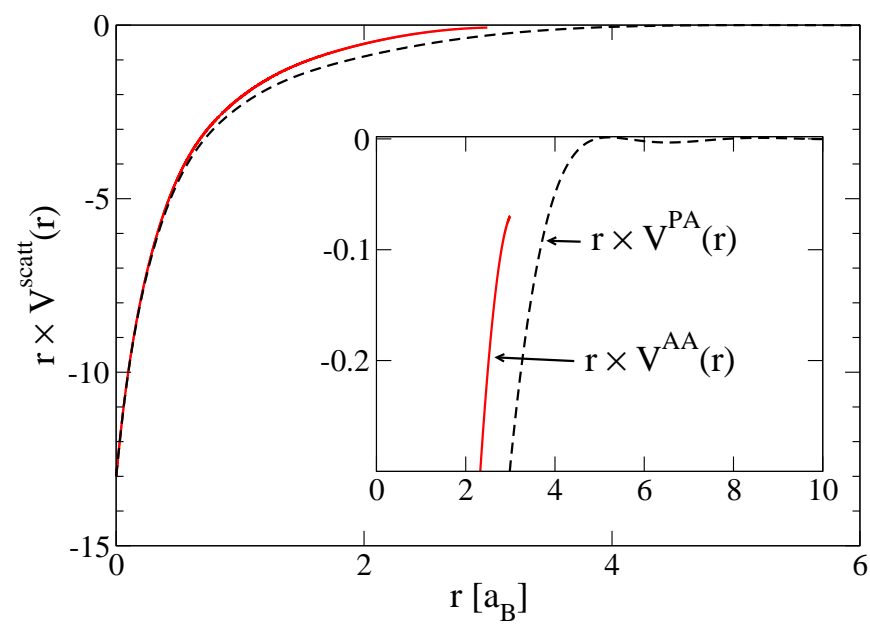

FIG. 2: (Color online) An example of the potentials $V^{A A}$ and $V^{P A}$ for aluminum at solid density and $1 \mathrm{eV}$. The muffintin like $V^{A A}$ extends as far as the ion-sphere radius $R_{W S}$ ( $\approx 3 a_{B}$ for aluminum at this density). The potential $V^{P A}$ is not muffin-tin like and can have long range structure due to Friedel oscillations in the electron density. The inset shows the same potentials, but focused on the regions where the potentials are close to 0 .

plasmas we consider do not have a free electron DOS. An example of the differences between a free electron DOS and a calculated DOS is given in figure 1 . The important point is that with the calculated DOS a different chemical potential, $\mu_{e}$ say, is needed to recover the correct number of valence electrons $\bar{Z}$. If we use (the more realistic) $\mu_{e}$ in the conductivity formulas then because of their implicit free electron DOS, a different electron density, $n_{e}^{0}$ say, results from equation (11). Thus we have two reasonable choices: either use $\bar{n}_{e}^{0}$ and $\bar{\mu}_{e}$, or $n_{e}^{0}$ and $\mu_{e}$. In what follows we test both choices. Finally we note that this ambiguity is brought about by the implicit free electron DOS in the conductivity formulas and would be resolved if a calculated DOS could be used in equations (8) and (9). However, we do not attempt this here.

\section{UNDERLYING PHYSICAL MODEL}

The Ziman-like formulas require inputs of $V^{\text {scatt }}(r)$, $n_{e}^{*}, \mu_{e}^{*}$ and $S_{I I}(k)$ from an underlying physical model. Numerous models have been used in the literature, and each can be coupled to the Ziman-like formulas in different ways, depending on what one chooses for the inputs. Here, for our underlying model we choose the recently developed Pseudo-atom molecular dynamics (PAMD) [26]. This model self consistently calculates the electronic and ionic structure, and it agrees well with generally accepted DFT-MD methods on equation of state, ionic transport and the structure factor itself $[26,35]$. Moreover, it agrees well with a recent X-ray scattering experiment that is sensitive to the ion-ion structure factor [36]. Thus we have confidence in the inputs that it generates for the Ziman-like formulas. In the next section we summarize PAMD with the aim of describing the various options for how it can be coupled to the Ziman-like formulas.

\section{A. Summary of pseudo-atom molecular dynamics}

In PAMD the electron density of the plasma $n_{e}(\boldsymbol{r})$ is approximated by a superposition of identical pseudoatom electron densities $n_{e}^{P A}(r)$ placed at each nuclear site $\boldsymbol{R}_{i}$

$$
n_{e}(\boldsymbol{r})=\sum_{i} n_{e}^{P A}\left(\left|\boldsymbol{r}-\boldsymbol{R}_{i}\right|\right)
$$

The total scattering potential is given by

$$
V^{t o t}(\boldsymbol{r})=\sum_{i} V_{e l}^{P A}\left(\left|\boldsymbol{r}-\boldsymbol{R}_{i}\right|\right)+V_{e e}^{x c}\left[n_{e}(\boldsymbol{r})\right]
$$

where

$$
V_{e l}^{P A}(r)=\frac{-Z}{r}+\int d^{3} r^{\prime} \frac{n_{e}^{P A}\left(r^{\prime}\right)}{\left|\boldsymbol{r}^{\prime}-\boldsymbol{r}\right|}
$$

$Z$ is the nuclear charge of the pseudo-atom, and $V_{e e}^{x c}$ is the exchange and correlation potential. The ionic positions $\boldsymbol{R}_{i}$ are generated in PAMD using classical molecular dynamics (MD), with a pseudo-atom pair interaction potential $V(r)$ being calculated directly from $n_{e}^{P A}(r)$. Alternatively, if one requires only knowledge of the ionion structure factor, $V(r)$ can be used in the so-called Ornstein-Zernike (OZ) equations. Solution of the OZ equations is much faster than using MD and is very accurate provided a good approximation for the bridge function is used ${ }^{1}$. We have used the $\mathrm{OZ}$ solution for all results presented here.

The calculation of $V(r)$ has been described in detail in refs. [27, 35], but it is relevant to summarize it here. One starts by defining an electron density $n_{e}^{s c r}(r)$, that is the component of $n_{e}^{P A}(r)$ due solely to valence (or screening) electrons. In Fourier space, $V(k)$ is given by

$$
V(k)=\frac{4 \pi}{k^{2}} \bar{Z}^{2}-n_{e}^{s c r}(k) \frac{C_{i e}(k)}{\beta}
$$

where $\bar{Z}=\int d^{3} r n_{e}^{s c r}(r)$ and $-C_{i e}(k) / \beta$ is often called a pseudopotential [13]. This pseudopotential is generated by requiring that in the linear response regime $-C_{i e}(k) / \beta$ is the potential that returns the density $n_{e}^{s c r}$, i.e.

$$
n_{e}^{s c r}(k)=-\frac{C_{i e}(k)}{\beta} \chi_{e}(k)
$$

\footnotetext{
${ }^{1}$ For the bridge function have used VMHNC for single species plasmas [37, 38] and HNC for mixtures [28], since VMHNC is not available. See [36] for further details.
} 


\begin{tabular}{cl}
\hline \hline Input choice Description \\
\hline$V^{\text {scatt }}(r)$ & \\
$V^{A A}$ & Equation (26). The muffin-tin like average atom potential. \\
$V^{P A}$ & Equation (27). The potential due to one pseudo-atom. \\
$V^{s c r}$ & Equation (25). The screened pseudopotential. \\
\hline$\mu_{e}^{*}, n_{e}^{*}$ & \\
$\mu_{e}, n_{e}^{0}$ & The "physical" quantities calculated in the average atom model. The correct number of valence \\
& electrons is returned if $\mu_{e}$ is used with the calculated, non-free electron density of states. \\
$\bar{\mu}_{e}, \bar{n}_{e}^{0}$ & The quantities corresponding the density of valence electrons if a free-electron density of states is used.
\end{tabular}

TABLE I: Input choices for the Ziman-like formulas that are tested here.

where $\chi_{e}$ is the response function of the electrons (see equation (17) of [35]). This type of pseudopotential has been used, along with Born approximation, to calculate resistivities with the Ziman-Evans formula [13, 32]. While this pseudopotential is weak by construction (due to the linear response relation), it is not designed to preserve the scattering properties of the full scattering potential $\left(V^{P A}(r)\right.$ in our case, see equation $\left.(27)\right)$. In the usual pseudopotential formulation (the one assumed by Ziman [29]) one tries to preserve the phase shifts of the full scattering potential, and modify the potential only near the nuclei. This is not guaranteed for $-C_{i e}(k) / \beta$ with potentially serious consequences for the resulting scattering cross sections. Nevertheless, we have implemented this calculation and show results using it in section IV. It is important to note that it is the screened pseudopotential $V^{s c r}(r)$ that is used in the Ziman-like formulas, where

$$
V^{s c r}(k)=-\frac{C_{i e}(k)}{\epsilon(k) \beta}
$$

where $\epsilon(k)$ is the dielectric function [13].

The process for calculating $n_{e}^{P A}(r)$ involves solving an average atom (AA) model. In this model, we use density functional theory to find the electron density $n_{e}^{f u l l}(r)^{2}$ in a spherically symmetric model system, in which a nucleus of charge $Z$ is placed at the origin and the remaining ions in the system are modeled as a continuous distribution represented by a Heaviside step function $\Theta\left(r-R_{W S}\right)$ at the Wigner-Seitz radius $R_{W S}$ (this is called the ionsphere model). A sphere of radius $R_{W S}$ surrounding the central nucleus is required to the charge neutral. The total average atom potential is given by

$$
\begin{aligned}
V^{A A}(r)= & -\frac{Z}{r}+\int d \boldsymbol{r}^{\prime} \frac{\left(n_{e}^{f u l l}\left(r^{\prime}\right)-n_{e}^{0} \Theta\left(r^{\prime}-R_{W S}\right)\right)}{\left|\boldsymbol{r}-\boldsymbol{r}^{\prime}\right|} \\
& +V_{e e}^{x c}\left[n_{e}^{f u l l}(r)\right]-V_{e e}^{x c}\left[n_{e}^{0}\right]
\end{aligned}
$$

\footnotetext{
2 We retain the notation used in [35] with the exception that $V_{N e}^{e f f}$ is renamed $V^{A A}$
}

where $n_{e}^{0}$ is the electron density in the field free region. The average atom chemical potential $\mu_{e}$ is determined when solving the model, and is related to $n_{e}^{0}$ by equation (11). It is a good estimation of the "physical" chemical potential, as it takes into account a non-free electron density of states (see figure 1).

$V^{A A}(r)$ can be thought of as a muffin-tin potential, in the sense that Evans [30] meant. It violates the condition that no-two muffin-tins overlap, however this may not be too serious a problem since such overlaps are routinely tolerated when using muffin-tin potentials for X-ray spectroscopy calculations [39]. However it is fair to point out that the average atom potential is not generated in the same way as muffin-tin potentials are usually generated [39].

The screened potential (25) and the average atom potential (26) have been widely used with the Ziman-Evans formula to calculate conductivities [13, 23, 24, 40, 41]. A third type of potential, that of a single pseudo-atom, has also been used $[1,13]$. It is important to note that while all these previous calculations have used potentials falling broadly into these three categories, differences in implementation details are important and have significant consequences on conductivity calculations. In PAMD we can define a pseudo-atom potential $V^{P A}(r)$ as

$$
V^{P A}(r)=V_{e l}^{P A}(r)+V_{x c}^{P A}(r)
$$

where the electrostatic part $V_{e l}^{P A}$ was defined in equation (22). The exchange and correlation part $V_{x c}^{P A}$ cannot be exactly separated from $V_{e e}^{x c}\left[n_{e}(\boldsymbol{r})\right]$ (equation (21)), but a reasonable approximation can be written down in analogy with the definition of the pseudo-atom electron density. $n_{e}^{P A}(r)$ is calculated from the average atom electron density $n_{e}^{\text {full }}(r)$ by removing the electron density found in a system with the same surrounding ions, but no central nucleus, $n_{e}^{e x t}(r)$ (see [35] for further details):

$$
n_{e}^{P A}(r)=n_{e}^{f u l l}(r)-n_{e}^{e x t}(r)
$$

Therefore we define

$$
V_{x c}^{P A}(r) \equiv V_{e e}^{x c}\left[n_{e}^{f u l l}(r)\right]-V_{e e}^{x c}\left[n_{e}^{e x t}(r)\right]
$$

An example of $V^{A A}(r)$ and $V^{P A}(r)$ is given in figure 2 . Note that $V^{A A}(r)$ does not go exactly to zero at $R_{W S}$ 


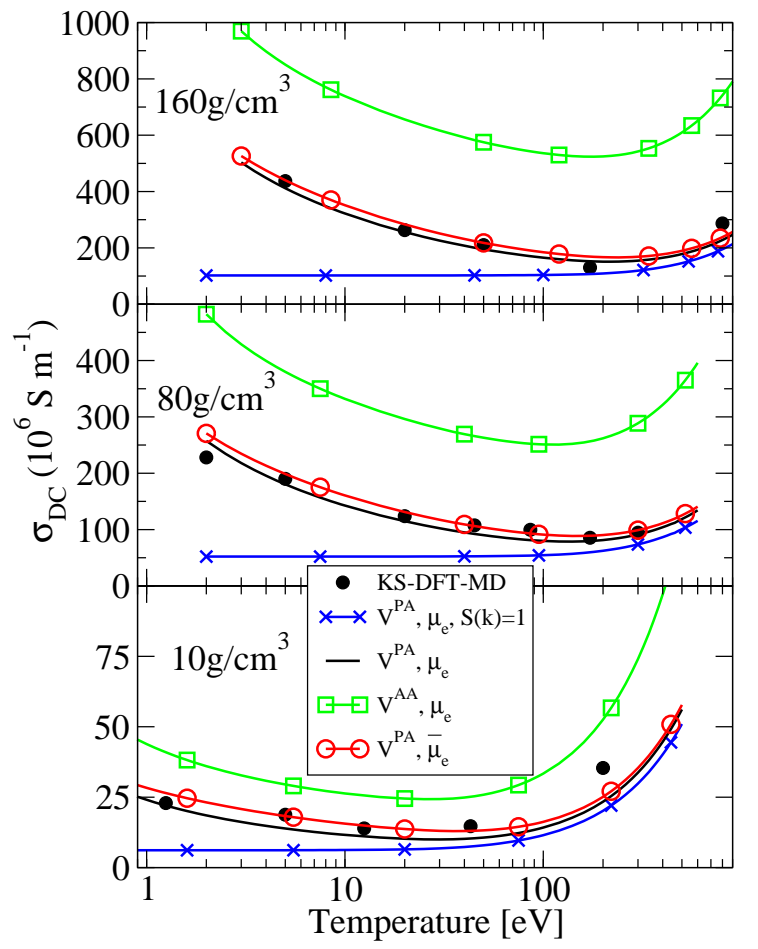

FIG. 3: (Color online) Electrical conductivity of dense hydrogen. All curves use PAMD-KS with a $t$-matrix calculation of the conductivity. The KS-DFT-MD data is from [2]. Clearly using the average atom (AA) potential grossly overestimates the conductivity relative to KS-DFT-MD, while setting $S(k)=1$ demonstrates that the increase in conductivity on lowering the temperature is due to changes in ionic structure.

(the muffin-tin radius), which is consistent with typical muffin-tin calculations [42].

In what follows we will numerically test all three potentials and both types of chemical potential. We will also demonstrate the effect of the ion-ion structure factor. All of these potentials, and the structure factor are self-consistently calculated in PAMD. Due to agreement with widely accepted DFT-MD simulations on equation of state, ionic self-diffusion and static ionic structure, as well as with available experimental measurements, we have a high degree of confidence in the inputs to the Ziman-like formulas generated by PAMD. Through a systematic comparison of these various inputs with KS-DFT-MD conductivity data we aim to evaluate the best choice of inputs, if such a choice does indeed exist. We restrict ourselves to comparisons where we have a high degree of confidence in the PAMD model. Roughly speaking this means temperatures $>1 \mathrm{eV}$ and densities roughly equal to or greater than solid density. It may be possible to go to lower densities or temperatures but that would require further testing and possible improvements to the PAMD model. As an aid to the reader we summarize the various input options that are to be tested in table I

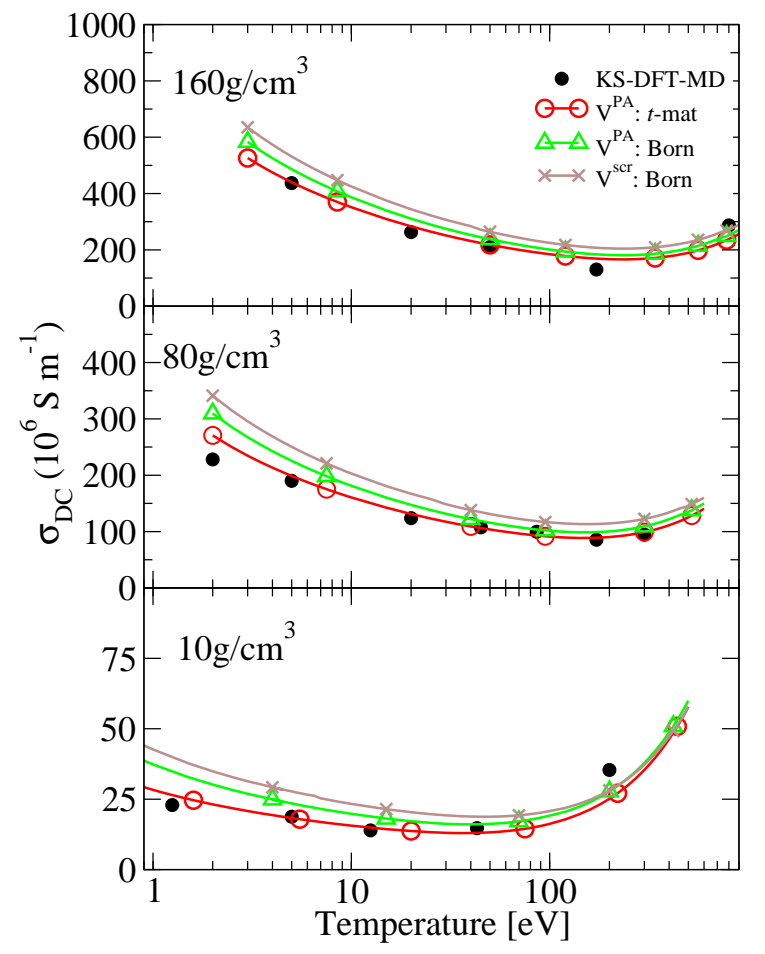

FIG. 4: (Color online) Electrical conductivity of dense hydrogen. All curves use PAMD-KS with chemical potential $\bar{\mu}_{e}$. The KS-DFT-MD data is from [2]. The Born calculations for both potentials are reasonably close to the KS-DFT-MD results.

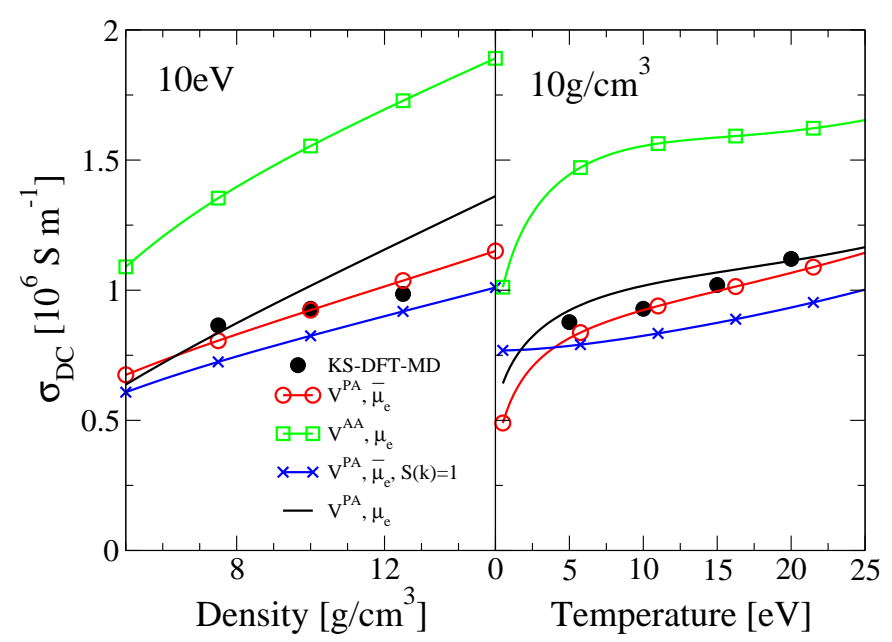

FIG. 5: (Color online) Electrical conductivity of dense beryllium. All curves use PAMD-KS with a $t$-matrix calculation of the conductivity. The KS-DFT-MD data is from [7]. As for hydrogen, using the average atom potential overestimates the conductivity. 


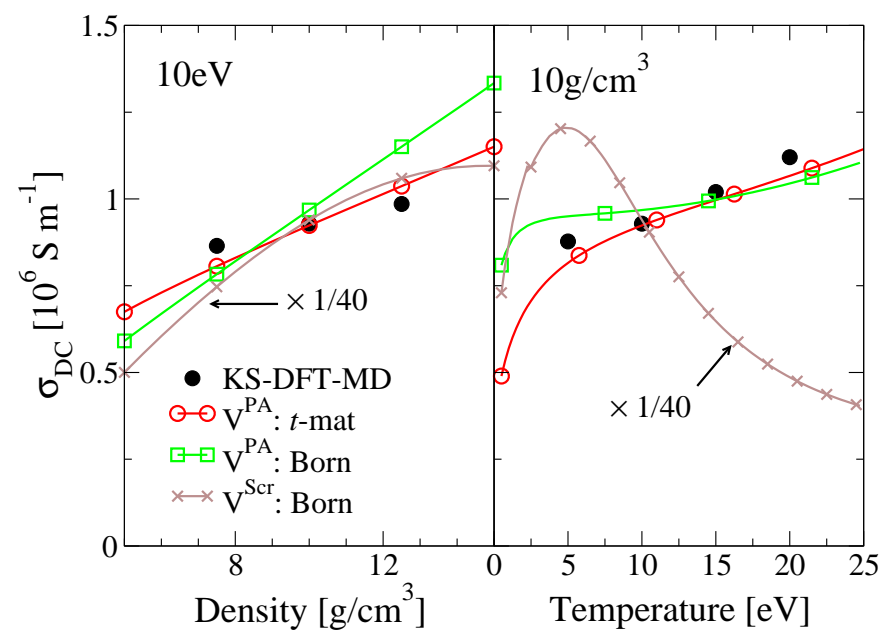

FIG. 6: (Color online) Electrical conductivity of dense beryllium. All curves use PAMD-KS with chemical potential $\bar{\mu}_{e}$. The KS-DFT-MD data is from [7]. While the Born approximation for the $V^{P A}$ potential gives reasonable results, that for $V^{s c r}$ strongly disagrees in magnitude with the KS-DFTMD data (it was scaled by a factor of $1 / 40$, as indicated in the plot).

\section{COMPARISON OF RESULTS}

PAMD is a density functional theory based model. In it we can use either Kohn-Sham (KS) or Thomas-Fermi (TF) treatments of the electronic kinetic energy term. The KS treatment is more physically accurate and in figures 3 to 10 we restrict ourselves to it. We must also choose an exchange and correlation potential, for which we have used the finite temperature local density approximation of [43]. Also, in figures 3 to 10 we use the inverse approach to conductivity only (equation (9)). We address Thomas-Fermi results and the direct approach in sections IVB and IV C. We compare to KS-DFTMD calculations and assume that these are close to the correct result. KS-DFT-MD is thought to be an accurate method for determining the electrical conductivity in warm dense matter, but is very expensive computationally. There can be issues with convergence of these calculations $[2,44]$ due to the extreme computational expense, but in the absence of experiments, they are widely accepted as being the most accurate data available.

In figure 3 we show the electrical conductivity of dense hydrogen. We have used the $t$-matrix method for all curves. Clearly, using the AA potential results in significantly too large a conductivity compared to KS-DFT$\mathrm{MD}$, though qualitatively the results with $V^{A A}$ are reasonable. Using the pseudo-atom potential $V^{P A}$ we find very good agreement with the KS-DFT-MD. For $V^{P A}$ we show results using both chemical potential options, and find only small differences. However, in the physical regimes shown in the plot, the two estimates of $n_{e}^{*}$ are close. Setting the structure factor to be equal to 1 , we

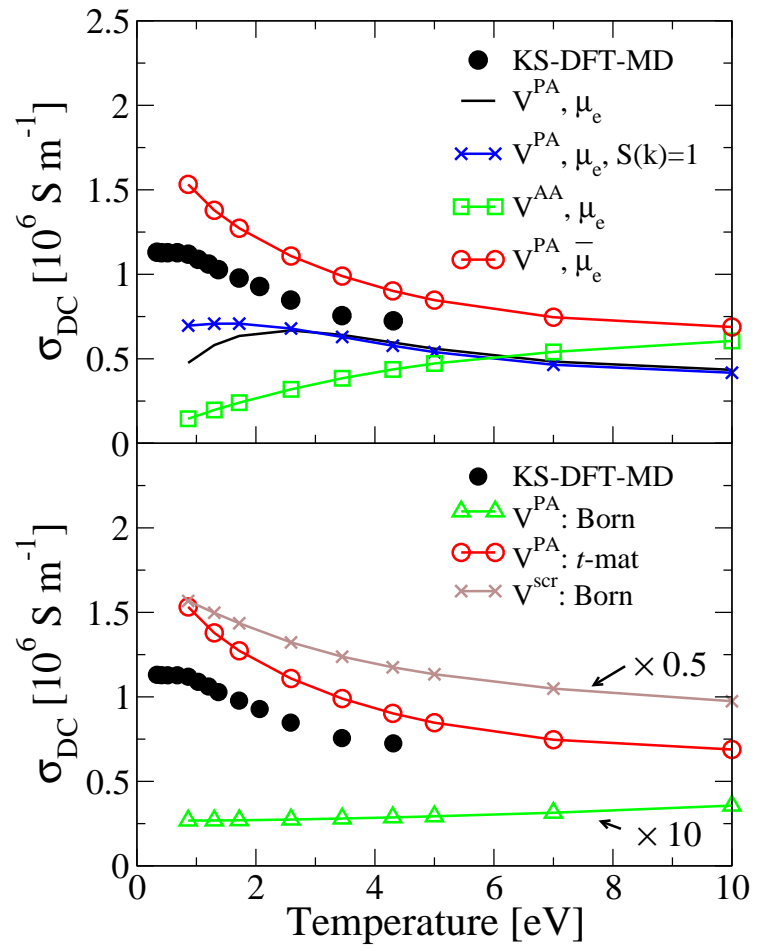

FIG. 7: (Color online) Electrical conductivity of molybdenum at $10 \mathrm{~g} / \mathrm{cm}^{3}$. All curves use PAMD-KS. In the top panel all calculations use the $t$-matrix method, in the bottom all calculations use the chemical potential $\bar{\mu}_{e}$. The KS-DFT-MD data is from [3]. In the bottom panel the $V^{s c r}$ Born results have been scaled by the factor indicated in the plot.

see that the rise in conductivity at low $\mathrm{T}$ is no longer reproduced. In [7] a similar effect was seen, though not explained. Those calculations [7] used an average atom potential and no explicit structure factor was included, thus implicitly using $S(k)=1$. From figure 3 we can now understand the result of [7]: The lack of the rise in conductivity at low $\mathrm{T}$ was due to the implicit $S(k)=1$, while the overestimation was because the average atom potential was used.

In figure 4 we show the same KS-DFT-MD hydrogen data, but now test the Born approximation. Using $V^{P A}$ with the Born approximation worsens agreement with the KS-DFT-MD data, though it is still reasonable. Using the screened pseudopotential $V^{s c r}$ with the Born approximation worsens the agreement still further.

We now turn to warm dense beryllium. This is a more complicated atom than hydrogen and one may expect a comparison to KS-DFT-MD data to be a more robust test of the method. In figure 5 we show $t$-matrix calculations. Again, we see that using $V^{A A}$ overestimates the KS-DFT-MD, while using $V^{P A}$ with either chemical potential choice gives good agreement. We find that for the conditions of the plot, setting $S(k)=1$ only has a small quantitative effect, though qualitatively a decrease in the conductivity at low $\mathrm{T}$ seen in the full calculation 


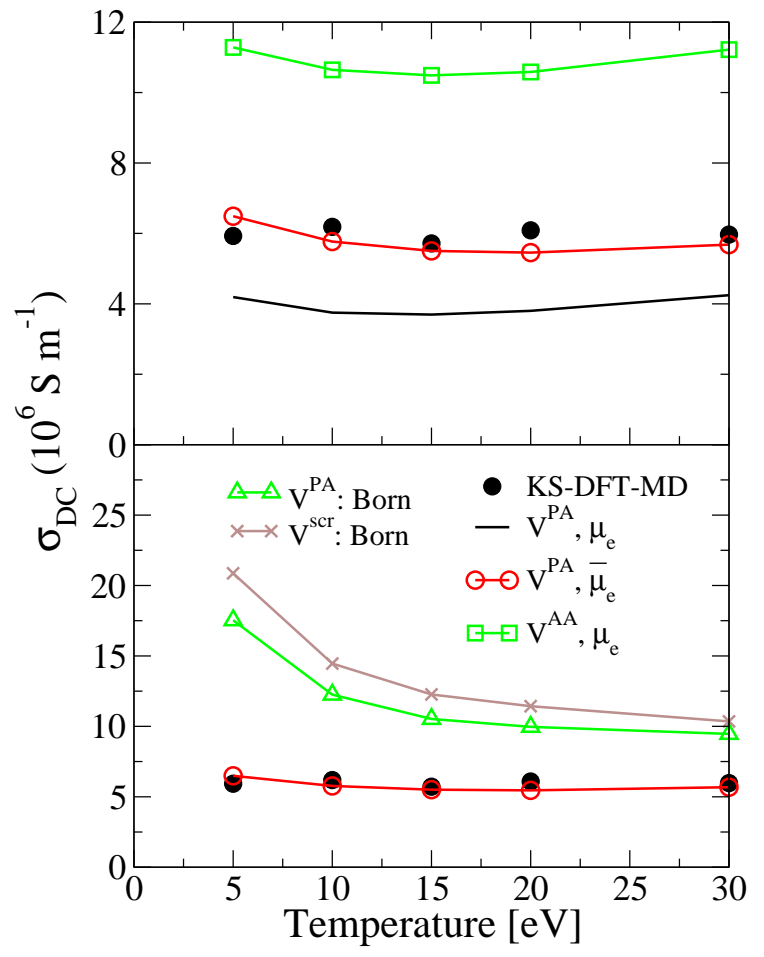

FIG. 8: (Color online) Electrical conductivity of a deuterium/tritium mixture $(1: 1)$ at $10 \mathrm{~g} / \mathrm{cm}^{3}$. All curves use PAMD-KS. In the top panel all calculations use the $t$-matrix method, in the bottom all calculations use the chemical potential $\bar{\mu}_{e}$. The KS-DFT-MD data is from [7].

disappears. In figure 6 we test the Born approximations. While using the Born approximation with $V^{P A}$ results in reasonable agreement with the KS-DFT-MD, using it with $V^{s c r}$ leads to vast differences. To fit the curve on the same plot we had to scale it by a factor of $1 / 40$. This result strongly suggests that, even where the Born approximation is reasonable, using the screened pseudopotential can lead to seriously inaccurate results.

Figure 7 shows results for molybdenum. This is a much more complicated atom than hydrogen or beryllium and is a transition metal. This comparison should be an even more stringent test of the method. In the figure see that both $V^{P A}$ and $V^{A A}$ give reasonable agreement in magnitude with the KS-DFT-MD. Using $V^{A A}$ gives poorer qualitative agreement at the lower temperatures, but we must be cautious, since it is likely that the underlying model (PAMD) will become less accurate at the lowest temperatures seen, where molybdenum approaches its solid phase. The two choices of chemical potential make a relatively large difference here, with the two curves bracketing the KS-DFT-MD data. The effect of $S(k)$ is only important at the lowest temperatures. In the bottom panel of the figure we test the Born approximation. For both potentials $V^{s c r}$ and $V^{P A}$ the results have been scaled as indicated, and both do a poor job of estimating the conductivity. Clearly the Born approx-

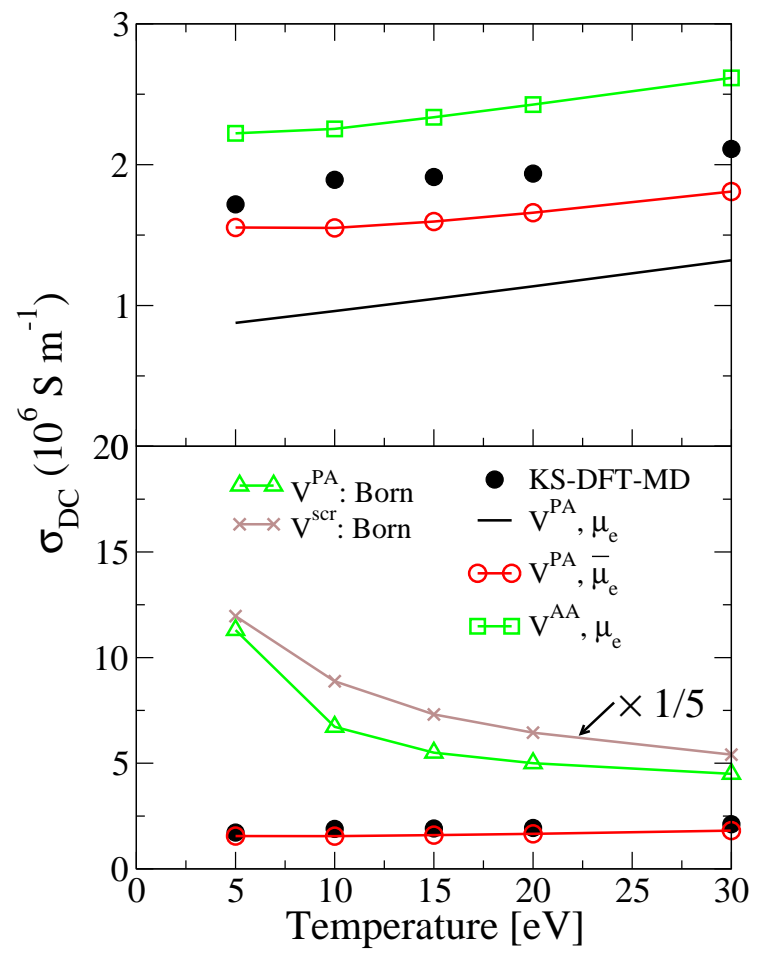

FIG. 9: (Color online) Electrical conductivity of a carbon/hydrogen mixture $(7: 9)$ at $10 \mathrm{~g} / \mathrm{cm}^{3}$. All curves use PAMD-KS. In the top panel all calculations use the $t$-matrix method, in the bottom all calculations use the chemical potential $\bar{\mu}_{e}$. The KS-DFT-MD data is from [7]. In the bottom panel the Born results have been scaled by the factors indicated in the plot.

imation has broken down here, as the large difference between the $t$-matrix and Born results for $V^{P A}$ indicate. The fact that using $V^{\text {scr }}$ comes closer to the data than for beryllium is probably due to a cancellation of errors: the Born approximation underestimates $\sigma_{D C}$, while $V^{s c r}$ leads to an overestimation.

We now turn to mixtures: deuterium/tritium (1:1), figure $8 ;$ carbon/hydrogen (7:9), figure 9 ; beryllium/deuterium/tritium (1:1:1), figure 10. The results are broadly in line with the previous result seen in figures 3 to 7 . Using $V^{P A}$ with either chemical potential gives good agreement with the KS-DFT-MD data, with the choice $n_{e}^{*}=\bar{n}_{e}^{0}$ leading to somewhat better agreement in general. Using $V^{A A}$ leads to an overestimation of the conductivity, though we find better agreement where the heavier element is present (the carbon/hydrogen mixture). In all cases the Born approximation with $V^{P A}$ overestimates the corresponding $t$-matrix calculation, and this overestimation becomes worse for the lower temperatures. Using $V^{s c r}$ with the Born approximation makes the agreement with KS-DFT-MD worse still, and for the carbon/hydrogen case it is in particularly bad agreement. 


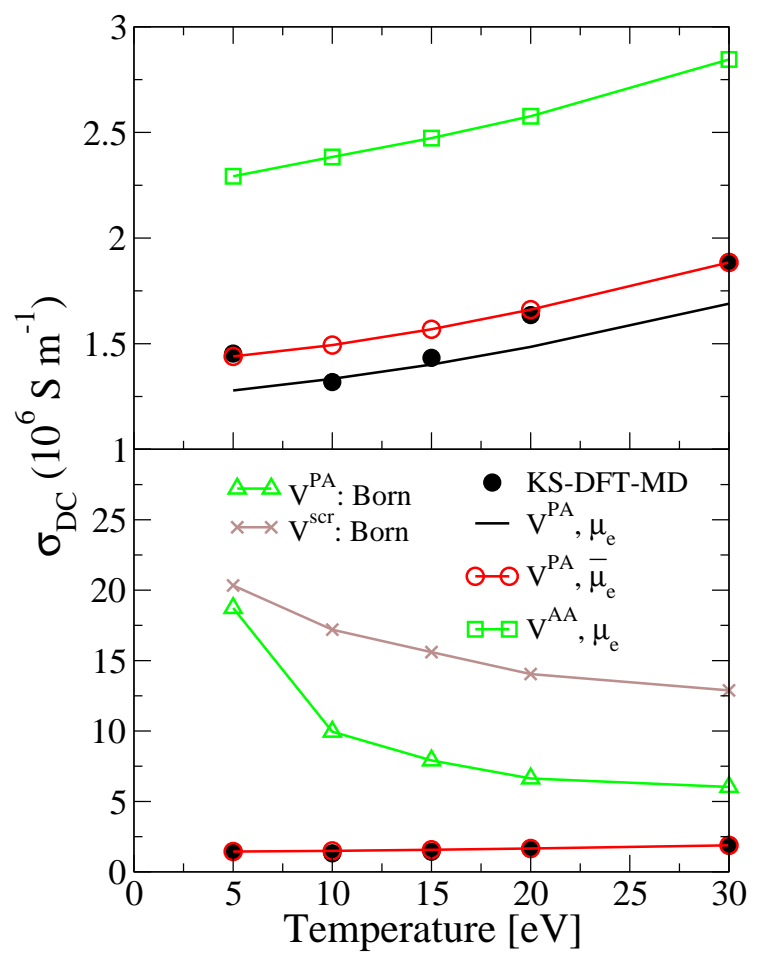

FIG. 10: (Color online) Electrical conductivity of a beryllium/deuterium/tritium mixture $(1: 1: 1)$ at $10 \mathrm{~g} / \mathrm{cm}^{3}$. All curves use PAMD-KS. In the top panel all calculations use the $t$-matrix method, in the bottom all calculations use the chemical potential $\bar{\mu}_{e}$. The KS-DFT-MD data is from [7].

\section{A. Discussion of the comparison to KS-DFT-MD}

The comparisons in figures 3 through 10 indicate that using $V^{P A}$ with either chemical potential option leads the good agreement with KS-DFT-MD data. Using $n_{e}^{*}=\bar{n}_{e}^{0}$ is perhaps the slightly better option, with significantly better agreement being found in some cases. While there is no phyical preference for choosing this option, our results indicate that the conductivity is less numerically sensitive to the choice of $n_{e}^{*}$ compared to the choice of $\mu_{e}^{*}$. Using $V^{s c r}$ with the Born approximation seems to be an unreliable method, sometimes leading to very inaccurate results, even where the Born approximation is reasonable. As discussed earlier, the screened pseudopotential is not designed for this purpose, and is not guaranteed to preserve the scattering properties of the full potential $\left(V^{P A}\right)$. Since $V^{s c r}$ is generated from the screening electron density $n_{e}^{s c r}(r)$, one possibility is that removing the oscillations in $n_{e}^{\text {scr }}(r)$ due to core valence orthogonality, before generating $V^{s c r}$, could improve the results. We have tested this by using the Troullier-Martins method [45], and found no significant improvement (not shown). Moreover, the resulting conductivity becomes strongly dependent on the Troullier-Martins core radius, a highly undesirable quality. In summary, we do not recommend using the $V^{s c r}$ potential.

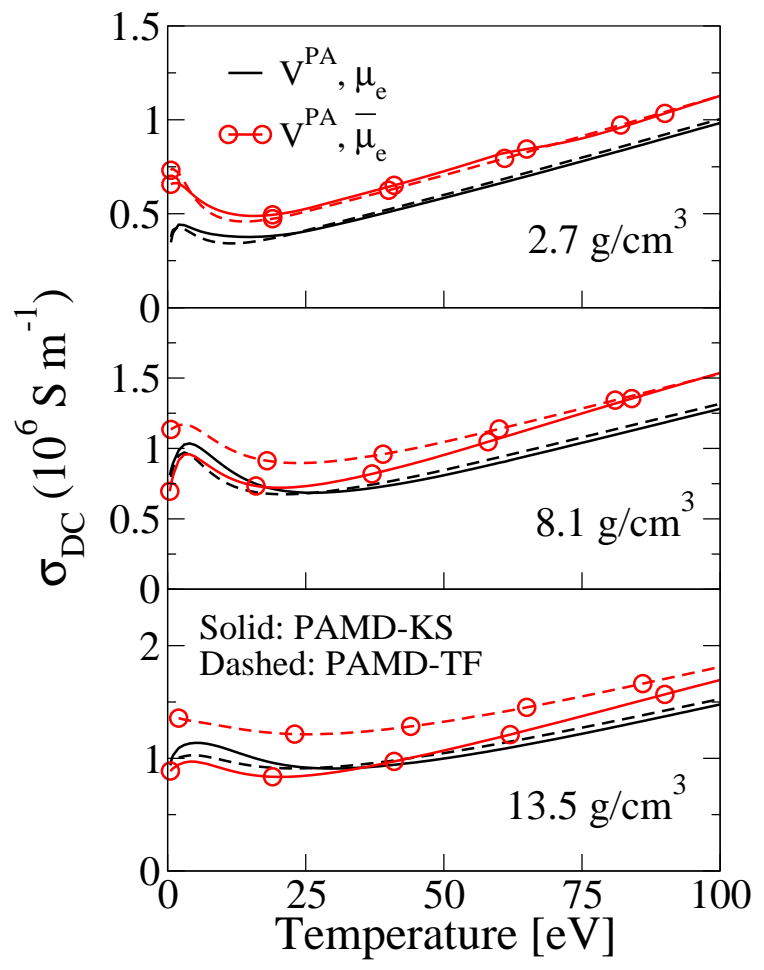

FIG. 11: (Color online) Electrical conductivity of aluminum, comparing Thomas-Fermi (TF) to Kohn-Sham (KS) based PAMD for three different densities. The PAMD-TF results are generally quite close to PAMD-KS, and are very computationally efficient to calculate. All calculations use $V^{P A}$ and the $t$-matrix approach.

The average atom potential leads to surprisingly poor agreement in magnitude for many cases. This is surprising because it has been used frequently in the past $[19,23,24]$, and because it is consistent with Evans' [30] original formulation. The failing may be due to the muffin-tin approximation. In making this approximation Evans's formula is limited to non-overlapping scattering potentials. By forcing the potential extend only as far as the muffin-tin radius (the Wigner-Seitz radius for $V^{A A}$ ) one immediately prevents recovery of Ziman's formula, which is correct in the Born approximation limit. In that limit, if the scattering potential is long ranged (i.e. longer ranged than $R_{W S}$ ) then the muffin-tin approximation will significantly change it (i.e. shorten its range). Only if the scattering potential is shorter ranged than $R_{W S}$ does Evans' approach recover Ziman's in the Born limit. Using $V^{P A}$ is a compromise between these two approaches that appears to capture the best parts of both. The potential is not modified by the muffin-tin approximation, so the Born limit can be recovered, while the use of the $t$-matrix method as proposed by Evans, allows strong scatterers to be present. Qualitatively, it is also consistent with Ziman's original idea of having isolated scattering centers [29]. 


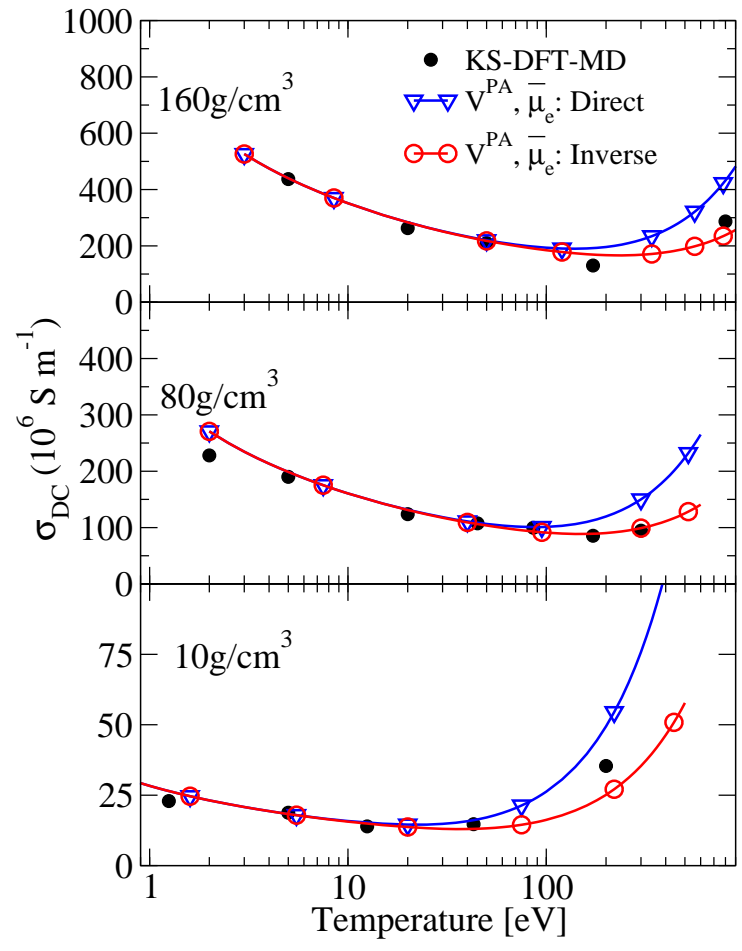

FIG. 12: (Color online) Electrical conductivity of warm dense hydrogen comparing the direct approach (equation (8)) to the inverse approach (equation (9)). All curves use PAMD-KS.

\section{B. Thomas-Fermi results}

As noted earlier, PAMD is a density functional theory based model. All results presented up to this point have used the Kohn-Sham (KS) functional. In figure 11 we compare conductivities for warm dense aluminum using KS to Thomas-Fermi (TF) calculations. All results use the $V^{P A}$ potential and the $t$-matrix approach. We show results from both chemical potential options. Generally the TF model is remarkably close to the KS based result. The results are closer when using $n_{e}^{0}$ compared to $\bar{n}_{e}^{0}$. This not surprising since the lack of atomic shell structure in TF leads to a continuous change in the number of valence electrons with density and temperature. Whereas, for KS the shell structure shows up as plateaus in the number of valence electrons. On the other hand the TF estimation of the chemical potential $\mu_{e}$ is generally quite close to the $\mathrm{KS}$ value, hence the closer agreement. Though TF is not as accurate a model as KS, it very computationally inexpensive, typically taking less than 1 minute per density and temperature point, compared to roughly 30-60 minutes for Kohn-Sham calculations on the same modern, desktop computer. It is therefore an attractive option for rapidly generating tables of reasonably accurate electrical conductivities. We note that $\mathrm{TF}$ based calculations for the other materials and conditions presented in figures 3 to 10 result in a similar level of agreement with corresponding KS calcu-

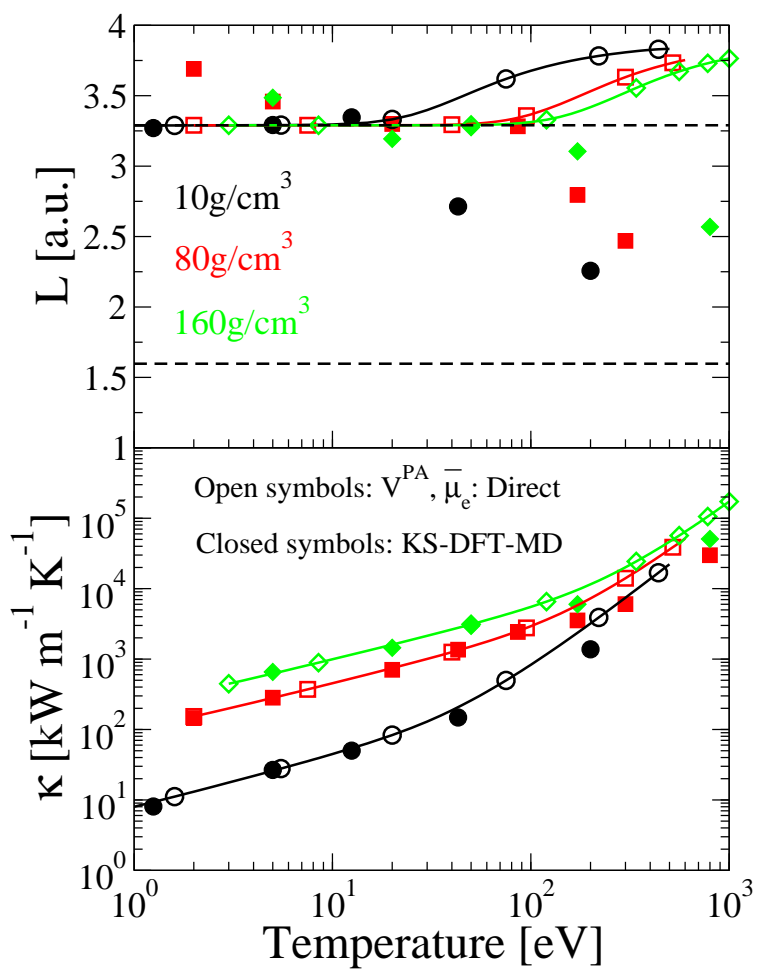

FIG. 13: (Color online) Thermal conductivity and Lorenz number of warm dense hydrogen using the direct approach (equation (8)). All curves use PAMD-KS. The horizontal dashed lines indicate the degenerate $(L=3.29$ a.u. $)$ and non-degenerate $(L=1.597$ a.u. $)$ limits.

lations (not shown).

\section{Direct approach and thermal conductivity}

In this section we compare the direct and inverse approaches to conductivity (equations (8) and (9)). We noted earlier that as $T \rightarrow 0$ both approaches give the same result, but at high $T$ the results will be different in general. In figure 12 we compare these approaches to KS-DFT-MD data for dense hydrogen. As expected both methods give similar answers for low temperature. At higher $T$ the direct approach gives much large values for the conductivity than the inverse approach. In comparison to the KS-DFT-MD data the inverse approach is generally closer, particularly at $80 \mathrm{~g} / \mathrm{cm}^{3}$. We have also checked the comparison to KS-DFT-MD for other cases (beryllium and molybdenum) but the results from the direct and inverse methods are similar due to the relatively low temperatures where KS-DFT-MD data is available.

In figure 13 we show the thermal conductivity and corresponding Lorenz number

$$
L=\frac{\kappa}{T \sigma_{D C}}
$$

calculated using the direct approach (see equation (16)). 
At low temperatures the agreement with KS-DFT-MD is excellent and the degenerate limit of the Lorenz number is reproduced. However, for higher temperatures the agreement with KS-DFT-MD becomes poor, and the direct approach results in an incorrect limit of the Lorenz number. Such behavior was also found in [7]. In that work the conductivities and Lorenz number were calculated in a similar approach, the major differences being that the structure factor was implicitly set to 1 and an average atom potential and chemical potential were used. We conclude that it is the direct approach, as opposed to the scattering potential, that is responsible for the incorrect limit of the Lorenz number. Therefore the direct approach must be treated with caution for higher temperatures where the plasma is transitioning to being non-degenerate.

\section{CONCLUSIONS}

Expressions for electron transport coefficients have been systematically tested by comparing to accurate but expensive Kohn-Sham denisty functional theory molecular dynamics (KS-DFT-MD) simulations. A number of implementation options have been tried, including choice of scattering potential, chemical potential and ion-ion structure factor, all of which are generated by a selfconsistent plasma model called pseudo-atom molecular dynamics [26]. For the scattering potential, three types of potential were tested: a muffin-tin like average atom potential; the potential due to one pseudo-atom (which is not muffin-tin like); and a pseudopotential method. We found that the pseudo-atom potential gives consistently good agreement with the KS-DFT-MD data for a range of elements (including mixtures), densities and temperatures. The average atom potential gives good qualitative agreement but can be significantly in error for magnitude. Finally, the pseudo-potential method was found to be unreliable, and the reasons for this are discussed. The effect of two reasonable choices of chemical potential was tested: first, a chemical potential that recovers the expected number of valence electrons with a free electron density of states $\left(\bar{\mu}_{e}\right)$; and second, a chemical potential that recovers the expected number of valence electrons with a calculated, non-free electron density of states $\left(\mu_{e}\right)$. The effect of the choice was generally quite modest, but using $\bar{\mu}_{e}$ generally results in better agreement with the KS-DFT-MD data.

Pseudo-atom molecular dynamics is a density functional theory based model and can therefore use different approximations for the electronic kinetic energy functional. We have compared the effect of using the accurate Kohn-Sham approximation to the less accurate, but very computationally efficient, Thomas-Fermi functional for warm dense aluminum. In general we find the Thomas-Fermi results are close to those using the KohnSham functional, and its use therefore allows very rapid generation of electron conductivities that are reasonably accurate.

Finally, we have tested an approach in which the conductivity is calculated directly and compared to the inverse approach, where the resistivity is directly calculated. Formally and numerically, both give similar answers for degenerate plasmas, but for higher temperatures where the plasma is transitioning to the nondegenerate regime, they give significantly different results. For dense hydrogen the inverse approach was found to give conductivities that agree better with KS-DFTMD data at high temperature. We used the direct approach to calculate the thermal conductivity and Lorenz number for warm dense hydrogen and found that, while good at low temperature in the degenerate regime, at higher temperatures the method does not tend to the correct non-degenerate limit, and significantly disagrees with KS-DFT-MD data. As a result we conclude that the direct approach should be used with caution in the non-degenerate regime.

\section{Acknowledgments}

We thank S. B. Hansen for useful conversations and suggestions. This work was performed under the auspices of the United States Department of Energy under contract DE-AC52-06NA25396 and LDRD number $20150656 \mathrm{ECR}$.
[1] Gérald Faussurier and Christophe Blancard. Resistivity saturation in warm dense matter. Phys. Rev. E, 91:013105, Jan 2015.

[2] Flavien Lambert, Vanina Recoules, Alain Decoster, Jean Clerouin, and Michael Desjarlais. On the transport coefficients of hydrogen in the inertial confinement fusion regime a). Physics of Plasmas (1994-present), 18(5):056306, 2011.

[3] Martin French and Thomas R Mattsson. Thermoelectric transport properties of molybdenum from ab initio simulations. Physical Review B, 90(16):165113, 2014.

[4] S. X. Hu, L. A. Collins, T. R. Boehly, J. D. Kress, V. N.
Goncharov, and S. Skupsky. First-principles thermal conductivity of warm-dense deuterium plasmas for inertial confinement fusion applications. Phys. Rev. E, 89:043105, Apr 2014.

[5] G. Faussurier, C. Blancard, P. Combis, and L. Videau. Electrical and thermal conductivities in dense plasmas. Physics of Plasmas (1994-present), 21(9):092706, 2014.

[6] H. Reinholz, G. Röpke, S. Rosmej, and R. Redmer. Conductivity of warm dense matter including electronelectron collisions. Physical Review E, 91(4):043105, 2015.

[7] C. E. Starrett, J Clérouin, V Recoules, J. D. Kress, 
L. A. Collins, and D. E. Hanson. Average atom transport properties for pure and mixed species in the hot and warm dense matter regimes. Physics of Plasmas (1994present), 19(10):102709, 2012.

[8] B. A. Hammel, S. W. Haan, D. S. Clark, M. J. Edwards, S. H. Langer, M. M. Marinak, and M. V. Patel. High energy density physics, 6:171, 2010.

[9] D. A. Greenwood. Proc. Phys. Soc. (London), 71:585, 1958.

[10] R. Kubo. J. Phys. Soc. (Japan), 12:570, 1957.

[11] D. E. Hanson, L. A. Collins, J. D. Kress, and M. P. Desjarlais. Phys. of. Plas., 18:082704, 2011.

[12] M. P. Desjarlais, J. D. Kress, and L. A. Collins. Electrical conductivity for warm, dense aluminum plasmas and liquids. Phys. Rev. E, 66:025401, Aug 2002.

[13] F. Perrot and M. W. C. Dharma-wardana. Phys. Rev. A, $36: 238,1987$.

[14] T. Blenski and K. Ishikawa. Phys. Rev. E, 51:4869, 1995.

[15] D. A. Liberman. Phys. Rev. B, 20:4981, 1979.

[16] G. Faussurier, C. Blancard, P. Cossé, and P. Renaudin. Phys. of. Plas., 17:052707, 2010.

[17] B. F. Rozsnyai. Phys. Rev. A, 5:1137, 1972.

[18] R. Piron and T. Blenski. Phys. Rev. E, 83:026403, 2011.

[19] P.A. Sterne, S.B. Hansen, B.G. Wilson, and W.A. Isaacs. High Energy Density Physics, 3:278, 2007.

[20] W. R. Johnson. High Energy Density Phys., 5:61, 2009.

[21] W. R. Johnson, C. Guet, and G. F. Bertsch. J. Quant. Spect. Rad. Trans., 99:327, 2006.

[22] M. Yu. Kuchiev and W. R. Johnson. Phys. Rev. E, 78:026401, 2008.

[23] J.C. Pain and G. Dejonghe. Electrical resistivity in warm dense plasmas beyond the average-atom model. Contributions to Plasma Physics, 50(1):39-45, 2010.

[24] Balazs F. Rozsnyai. Electron scattering in hot/warm plasmas. High Energy Density Physics, 4(1):64-72, 2008.

[25] M. W. C. Dharma-wardana. Static and dynamic conductivity of warm dense matter within a density-functional approach: Application to aluminum and gold. Phys. Rev. E, 73:036401, Mar 2006

[26] C. E. Starrett, J. Daligault, and D. Saumon. Phys. Rev. E, 91:013104, 2015.

[27] C. E. Starrett and D. Saumon. Phys. Rev. E, 87:013104, 2013.
[28] C. E. Starrett, D. Saumon, J. Daligault, and S. Hamel. Phys. Rev. E, 90:033110, 2014.

[29] J. M. Ziman. Philos. Mag., 6:1013, 1961.

[30] R. Evans, B. L. Gyorfey, N. Szabo, and J. M. Ziman. On the resistivity of liquid transition metals. In S. Takeuchi, editor, The properties of liquid metals. Taylor and Francis, London, 1973.

[31] Thomas E Faber. Introduction to the theory of liquid metals. Cambridge University Press, 1972.

[32] Setsuo Ichimaru and Shigenori Tanaka. Theory of interparticle correlations in dense, high-temperature plasmas. v. electric and thermal conductivities. Phys. Rev. A, 32:1790-1798, Sep 1985.

[33] F. Perrot and M. W. C. Dharma-wardana. Phys. Rev. E, 52:5352, 1995.

[34] John M Ziman. Electrons and phonons: the theory of transport phenomena in solids. Oxford University Press, 1960.

[35] C. E. Starrett and D. Saumon. High Energy Dens. Phys., 10:35, 2014

[36] C. E. Starrett and D. Saumon. Models of the elastic xray scattering feature for warm dense matter. Phys. Rev. E, 92:033101, 2015.

[37] G. Faussurier. Phys. Rev. E, 69:066402, 2004.

[38] Y. Rosenfeld. J. Stat. Phys., 42:437, 1986.

[39] C. R. Natoli, M. Benfatto, S. Della Longa, and K. Hatada. X-ray absorption spectroscopy: state-of-theart analysis. Journal of synchrotron radiation, 10(1):2642, 2003.

[40] George A. Rinker. Systematic calculations of plasma transport coefficients for the periodic table. Phys. Rev. A, 37:1284-1297, 1988.

[41] S. B. Hansen, D. A. Young, B. G. Wilson, P. A. Sterne, W. A. Isaacs, and V. Sonnad. Electrical conductivity calculations from the Purgatorio code. United States. Department of Energy, 2006.

[42] O. Peyrusse. J. Phys: condensed matter, 20:195211, 2008.

[43] Valentin V. Karasiev, Travis Sjostrom, James Dufty, and S. B. Trickey. Phys. Rev. Lett., 112:076403, 2014.

[44] V. Recoules, F. Lambert, A. Decoster, B. Canaud, and J. Clérouin. Phys. Rev. Lett., 102:075002, 2009.

[45] N. Troullier and J. L. Martins. Phys. Rev. B, 43:1993, 1991. 